NASA Contractor Report 182209

AIAA-88-0467

\title{
A Diagonally Inverted LU Implicit Multigrid Scheme for the 3-D Navier-Stokes Equations and a Two Equation Model of Turbulence
}

Jeffrey W. Yokota

Sverdrup Technology, Inc.

NASA Lewis Research Center Group

Cleveland, Ohio

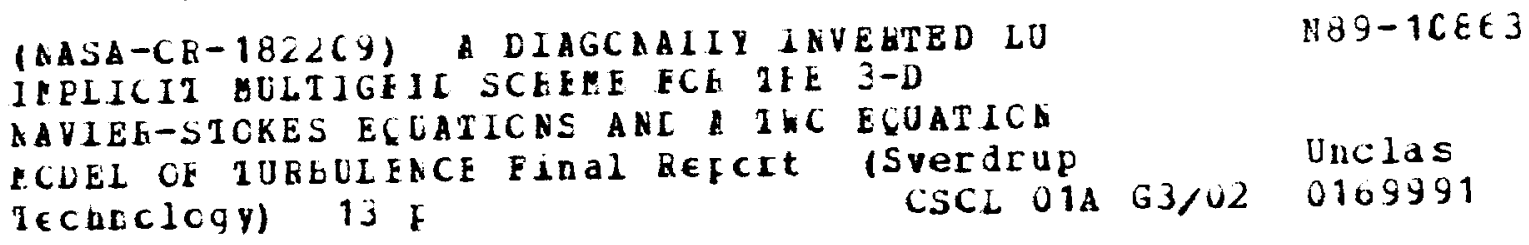

October 1988

Prepared for

Lewis Research Center

Under Contract NAS3-25266

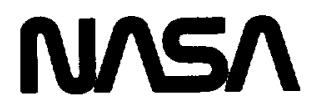

National Aeronautics and

Space Administration 


\title{
A Diagonally Inverted LU Implicit Multigrid Scheme \\ for the 3-D Navier-Stokes Equations and \\ a Two Equation Model of Turbulence
}

\author{
Jeffrey W. Yokota \\ Sverdrup Technology, Inc. \\ NASA Lewis Research Center Group \\ Cleveland, Ohio 44135
}

\begin{abstract}
An LU implicit multigrid algorithm is developed to calculate three-dimensional compressible viscous flows. This scheme solves the full three-dimensional ReynoldsAveraged Navier-Stokes equation with a two equation $k-\varepsilon$ model of turbulence. The flow equations are integrated by an efficient, diagonally inverted, LU implicit multigrid scheme while the $k-\epsilon$ equations are solved, uncoupled from the flow equations, by a block LU implicit algorithm. The flow equations are solved within the framework of the multigrid method using a four grid level W-cycle, while the $k-\epsilon$ equations are iterated only on the finest grid. This treatment of the Reynolds-Averaged Navier-Stokes equations proves to be an efficient method for calculating threedimensional compressible viscous flows.
\end{abstract}

\section{Introduction}

As the availability of larger and more powerful computers increases, so will the attention being directed towards computing complex three-dimensional compressible viscous Hows. At present the most sucessful approach has been to solve the Thin-Layer Navier-Stokes equations with an algebraic turbulence model. Most notable has been the work of Pulliam and Steger ${ }^{1}$ and their highly sucessful ARC codes.

Attempts to produce efficient numerical schemes for the calculation of compressible viscous flows have led to the Diagonalized ADI scheme of Chaussee and Pulliam ${ }^{2}$ and more recently to the multigrid Runge-Kutta scheme of Jayaram and Jameson. ${ }^{3}$ As emphasis increasingly focuses upon calculating complex three-dimensional flows, the need to numerically solve the Reynolds-Averaged Navier-Stokes equations efficiently is correspondingly apparent.

The present work deals with the development of an efficient LU implicit multigrid scheme for the numerical solution of the Reynolds-Averaged Navier-Stokes equations and the two equation $k-\epsilon$ turbulence model. The ReynoldsAveraged Navier-Stokes equations, which for mathematical closure require the modelling of the Reynolds stress tensor, are solved by the Diagonally Inverted LU Implicit Multigrid scheme developed by Yokota, Caughey, and Chima." A Boussinesq eddy-viscosity formulation is used to model the Reynolds stress term, where the turbulent viscosities are calculated from a standard high Reynolds number $k-\varepsilon$ model.

\section{Analysis}

The three-dimensional Reynolds-Averaged NavierStokes equations, with the Boussinesq eddy-viscosity formulation, are written in divergence form and then transformed from the Cartesian coordinate system $(x, y, z)$ to the generalized system $(\xi, \eta, \xi)$. The resulting equations can be written:

$$
\begin{gathered}
\frac{\partial \vec{W}}{\partial t}+\frac{\partial \vec{F}}{\partial \xi}+\frac{\partial \vec{G}}{\partial \eta}+\frac{\partial \vec{H}}{\partial \zeta}= \\
\frac{\partial \vec{F}_{u}}{\partial \xi}+\frac{\partial \vec{G}_{v}}{\partial \eta}+\frac{\partial \vec{H}_{v}}{\partial \zeta}
\end{gathered}
$$

with

$$
\begin{aligned}
& \vec{W}=\left(\begin{array}{c}
\rho D \\
\rho D u \\
\rho D v \\
\rho D w \\
\rho D E
\end{array}\right) \\
& \vec{F}=\left(\begin{array}{c}
\rho D U \\
\rho D U u+P D \xi_{x} \\
\rho D U v+P D \xi_{y} \\
\rho D U w+P D \xi_{x} \\
D U(E+P)
\end{array}\right) \\
& \vec{G}=\left(\begin{array}{c}
\rho D V \\
\rho D V u+P D \eta_{x} \\
\rho D V v+P D \eta_{y} \\
\rho D V u+P D \eta_{z} \\
D V(E+P)
\end{array}\right) \\
& \vec{H}=\left(\begin{array}{c}
\rho D W \\
\rho D W u+P D_{\zeta_{x}} \\
\rho D W v+P D_{\zeta_{y}} \\
\rho D W w+P D_{\zeta_{z}} \\
D W(E+P)
\end{array}\right) \\
& \vec{F}_{v}=\left(\begin{array}{l}
f_{v_{1}} \\
f_{v_{2}} \\
f_{v_{3}} \\
f_{v_{4}} \\
f_{v_{3}}
\end{array}\right)= \\
& \left(\begin{array}{c}
0 \\
D \xi_{x} \tau_{x x}+D \xi_{y} \tau_{x y}+D \xi_{z} \tau_{x z} \\
D \xi_{x} \tau_{x y}+D \xi_{y} \tau_{y y}+D \xi_{x} \tau_{y x} \\
D \xi_{x} \tau_{x z}+D \xi_{y} \tau_{y z}+D \xi_{x} \tau_{z x} \\
u f_{v_{2}}+v f_{v s}+w f_{v_{1}}+\frac{\gamma}{\gamma-1} \tilde{\mu}_{e}\left(c_{1} \frac{\partial T}{\partial \xi}+c_{2} \frac{\partial T}{\partial \eta}+c_{3} \frac{\partial T}{\partial \xi}\right)
\end{array}\right) \\
& \vec{G}_{v}=\left(\begin{array}{l}
g_{v_{1}} \\
g_{v_{2}} \\
g_{v_{3}} \\
g_{v_{1}} \\
g_{v_{3}}
\end{array}\right)=
\end{aligned}
$$


where

$$
\begin{aligned}
& \vec{W}_{k \varepsilon}=\left(\begin{array}{c}
\rho D k \\
\rho D \epsilon
\end{array}\right) \quad \vec{S}_{k \varepsilon}=\left(\begin{array}{c}
s_{1} \\
s_{2}
\end{array}\right) \\
& \vec{F}_{k \varepsilon}=\left(\begin{array}{l}
\rho D U k-\mu_{k}\left(c_{1} \frac{\partial k}{\partial \xi}+c_{2} \frac{\partial k}{\partial \eta}+c_{3} \frac{\partial k}{\partial \zeta}\right) \\
\rho D U \varepsilon-\mu_{c}\left(c_{1} \frac{\partial \varepsilon}{\partial \xi}+c_{2} \frac{\partial \varepsilon}{\partial \eta}+c_{3} \frac{\partial \varepsilon}{\partial \zeta}\right)
\end{array}\right) \\
& \vec{G}_{k \varepsilon}=\left(\begin{array}{c}
\rho D V k-\mu_{k}\left(c_{4} \frac{\partial k}{\partial \xi}+c_{5} \frac{\partial k}{\partial \eta}+c_{6} \frac{\partial k}{\partial \varsigma}\right) \\
\rho D V \varepsilon-\mu_{6}\left(c_{4} \frac{\partial \varepsilon}{\partial \xi}+c_{5} \frac{\partial \varepsilon}{\partial \eta}+c_{6} \frac{\partial \varepsilon}{\partial \varsigma}\right)
\end{array}\right) \\
& \vec{H}_{k \varepsilon}=\left(\begin{array}{c}
\rho D W k-\mu_{k}\left(c_{7} \frac{\partial k}{\partial \xi}+c_{8} \frac{\partial k}{\partial \eta}+c_{9} \frac{\partial k}{\partial \zeta}\right) \\
\rho D W \varepsilon-\mu_{\varepsilon}\left(c_{7} \frac{\partial \varepsilon}{\partial \xi}+c_{8} \frac{\partial \varepsilon}{\partial \eta}+c_{9} \frac{\partial c}{\partial \zeta}\right)
\end{array}\right)
\end{aligned}
$$

and

$$
\begin{aligned}
s_{1} & =D(\Theta-\rho \varepsilon) \\
s_{2} & =\frac{D\left(t_{1} \Theta-t_{2} \rho \epsilon\right) \epsilon}{k} \\
\mu_{k} & =\mu_{l}+\frac{\mu_{t}}{\sigma_{k}} \\
\mu_{\varepsilon} & =\mu_{l}+\frac{\mu_{t}}{\sigma_{\varepsilon}}
\end{aligned}
$$

for which the production rate of the turbulence kinetic energy is defined as

$$
\begin{aligned}
\Theta & =\left(2 \mu_{t} \frac{\partial u}{\partial x}-\frac{2}{3}\left(\rho k+\mu_{t}\left(\frac{\partial u}{\partial x}+\frac{\partial v}{\partial y}+\frac{\partial w}{\partial z}\right)\right)\right) \frac{\partial u}{\partial x} \\
& +\left(2 \mu_{t} \frac{\partial v}{\partial y}-\frac{2}{3}\left(\rho k+\mu_{t}\left(\frac{\partial u}{\partial x}+\frac{\partial v}{\partial y}+\frac{\partial w}{\partial z}\right)\right)\right) \frac{\partial v}{\partial y} \\
& +\left(2 \mu_{t} \frac{\partial w}{\partial z}-\frac{2}{3}\left(\rho k+\mu_{t}\left(\frac{\partial u}{\partial x}+\frac{\partial v}{\partial y}+\frac{\partial w}{\partial z}\right)\right)\right) \frac{\partial w}{\partial z} \\
& +\mu_{t}\left(\frac{\partial u}{\partial y}+\frac{\partial u}{\partial z}+\frac{\partial v}{\partial x}+\frac{\partial v}{\partial z}+\frac{\partial w}{\partial x}+\frac{\partial w}{\partial y}\right)^{2}
\end{aligned}
$$

The turbulence modelling constants are chosen to be the standard Launder and Spalding ${ }^{9}$ values of

$$
\begin{gathered}
C_{\mu}=0.09 \quad t_{1}=1.44 \quad t_{2}=1.92 \\
\sigma_{k}=1.0 \quad \sigma_{\star}=1.3
\end{gathered}
$$

and were never altered during the course of this work. (Isotropy is assumed since $C_{\mu}$ is a scalar constant rather than a non-constant vector quantity.) The transformed Navier-Stokes and $k-\varepsilon$ equations are discretized by a finite volume formulation that approximates the spatial differences as a net fux across the faces of each mesh cell. Global conservation and the admission of possible uniform flow solutions are insured by evaluating both the inviscid and viscous flux vectors on the faces of the boundary-conforming mesh cells. This procedure requires that the flow and $k-\varepsilon$ variables, viscous stresses, and flux-embedded geometric quantities be defined on the faces of the mesh cells during the flux evaluations, although it is the cell-averaged flow and $k-\epsilon$ variables that are calculated during the time and spatial marching. The viscous stresses and geometric quantities are evaluated directly on the cell faces, while the flow and $k-\epsilon$ variables are averaged over values found in adjacent cells. The unsteady equations can be discretized into an implicit approximation that, when written in a linearized delta form, produces a numerical scheme whose steady state solutions are independent of the time step size used in the time marching. The computational effort required to construct this implicit approximation is kept at a minimum by treating only the inviscid fluxes. The delta form, produced by linearizing the changes in the inviscid flux vectors through a Taylor series expansion about a time level $n$, can be written for the flow equations as follows:

$$
\begin{gathered}
{\left[I+\mu_{i} \Delta t\left(\delta_{\xi} A+\delta_{\eta} B+\delta_{\xi} C\right)\right] \Delta \vec{W}_{i j k}^{n}=} \\
-\Delta t\left(\bar{\delta}_{\xi}\left(\vec{F}-\vec{F}_{v}\right)+\bar{\delta}_{\eta}\left(\vec{G}-\vec{G}_{v}\right)+\vec{\delta}_{\zeta}\left(\vec{H}-\vec{H}_{v}\right)\right)_{i j k}^{n}
\end{gathered}
$$

where

$$
\Delta \vec{W}^{n}=\vec{W}^{n+1}-\vec{W}^{n}
$$

and $\Delta t$ is the time step size; $0 \leq \mu_{i} \leq 1$ is a parameter governing the degree of implicitness; $\delta$ and $\delta$ are cell- and face-centered central differences; $I$ is the identity matrix; and $A, B$, and $C$ are the inviscid flux Jacobian matrices relative to the vectors $\vec{F}, \vec{G}$, and $\vec{H}$. The implicit form of the $k-\epsilon$ equations can be written as follows:

$$
\begin{aligned}
& {\left[I+\mu_{i} \Delta t\left(\delta_{\xi} A_{k s}+\delta_{\eta} B_{k \epsilon}+\delta_{\xi} C_{k s}-E_{k c}\right)\right] \Delta \vec{W}_{k s}^{n} l_{i j k}=} \\
& -\Delta t\left(\vec{\delta}_{\xi} \vec{F}_{k \varepsilon}+\bar{\delta}_{\eta} \vec{G}_{k \varepsilon}+\bar{\delta}_{\xi} \vec{H}_{k \varepsilon}-\vec{S}_{k \varepsilon}\right)_{i j k}^{n}
\end{aligned}
$$

and

$$
\Delta \vec{W}_{k s}^{n}=\vec{W}_{k+}^{n+1}-\vec{W}_{k s}^{n}
$$

where $A_{k e}, B_{k c}$, and $C_{k c}$ are the inviscid flux Jacobian matrices relative to the inviscid terms found in the vectors $\vec{F}_{k e}, \vec{G}_{k e}$, and $\vec{H}_{k r} ;$ and $E_{k,}$ is the Jacobian matrix relative to the source vector $\vec{S}_{k e}$.

\section{Artificial Dissipation}

The finite volume formulation reduces to a central difference approximation on a uniform grid and therefore requires the addition of explicit artificial dissipation terms 
2) Upper Sweep

$$
\left[I+\mu_{i} \Delta t\left(\delta_{t}^{+} A_{2}+\delta_{\eta}^{+} B_{2}+\delta_{\zeta}^{+} C_{2}\right)\right] \Delta \vec{W}_{i j k}^{n}=\Delta \vec{Y}_{i j k}^{n}
$$

A similar explicit sweep, but in directions opposite to those taken in the lower sweep, is needed to solve this system of equations. The resulting flow field corrections are then used to update the flow field.

$$
\vec{W}_{i j k}^{n+1}=\Delta \vec{W}_{i j k}^{n}+\vec{W}_{i j k}^{n}
$$

The LU factorization requires the solution of two block triangular operators each of which, through back substitution, can be reduced to simple $5 \times 5$ matrix systems at every mesh cell. These reduced systems can be written:

1) Lower Sweep

$$
\begin{gathered}
{\left[I+\mu_{i} \Delta t\left(A_{1}+B_{1}+C_{1}\right)\right] \Delta \vec{Y}_{i j k}^{n}=} \\
-\Delta t\left(\bar{\delta}_{\xi}\left(\vec{F}-\vec{F}_{v}\right)+\bar{\delta}_{\eta}\left(\vec{G}-\vec{G}_{v}\right)+\bar{\delta}_{s}\left(\vec{H}-\vec{H}_{v}\right)+\vec{T}_{i j k}^{n}\right. \\
+\mu_{i} \Delta t\left(A_{1} \Delta \vec{Y}_{i-1, j, k}^{n}+B_{1} \Delta \vec{Y}_{i, j-1, k}^{n}+C_{1} \Delta \vec{Y}_{i, j, k-1}^{n}\right)
\end{gathered}
$$

2) Upper Sweep

$$
\begin{gathered}
{\left[I-\mu_{i} \Delta t\left(A_{2}+B_{2}+C_{2}\right)\right] \Delta \vec{W}_{i j k}^{n}=} \\
\Delta \vec{Y}_{i j k}^{n}-\mu_{i} \Delta t\left(A_{2} \Delta \vec{W}_{i+1, j, k}^{n}\right. \\
\left.+B_{2} \Delta \vec{W}_{i, j+1, k}^{n}+C_{2} \Delta \vec{W}_{i, j, k+1}^{n}\right)
\end{gathered}
$$

A similar analysis for the $k-\varepsilon$ equations can be used to write the following $2 \times 2$ matrix equations:

1) Lower Sweep

$$
\begin{aligned}
& {\left.\left[I+\mu_{i} \Delta t\left(A_{k \varepsilon_{1}}+B_{k \varepsilon_{1}}+C_{k \varepsilon_{1}}-E_{k \varepsilon_{1}}\right)\right] \Delta \vec{Y}_{k s}\right|_{i j k} ^{n}=} \\
& -\Delta t\left(\bar{\delta}_{\xi} \vec{F}_{k \varepsilon}+\bar{\delta}_{\eta} \vec{G}_{k \varepsilon}+\bar{\delta}_{\xi} \vec{H}_{k \varepsilon}-E_{k \varepsilon}+\vec{T}_{k \varepsilon}\right)_{i j k}^{n} \\
& +\mu_{i} \Delta t\left(\left.A_{k f_{1}} \Delta \vec{Y}_{k \boldsymbol{r}}\right|_{i-1, j, k} ^{n}+\left.B_{k \epsilon_{1}} \Delta \vec{Y}_{k \boldsymbol{r}}\right|_{i, j-1, k} ^{n}\right. \\
& \left.+\left.C_{k a_{1}} \Delta \vec{Y}_{k \sigma}\right|_{i, j, k-1} ^{n}\right)
\end{aligned}
$$

2) Upper Sweep

$$
\begin{gathered}
{\left.\left[I-\mu_{i} \Delta t\left(A_{k \varepsilon_{3}}+B_{k s_{2}}+C_{k \varepsilon_{3}}-E_{k \varepsilon_{1}}\right)\right] \Delta \vec{W}_{k \varepsilon}\right|_{i j k} ^{n}=} \\
\left.\Delta \vec{Y}_{k \varepsilon}\right|_{i j k} ^{n}-\mu_{i} \Delta t\left(\left.A_{k \varepsilon_{3}} \Delta \vec{W}_{k \varepsilon}\right|_{i+1, j, k} ^{n}\right. \\
\left.+\left.B_{k \varepsilon_{2}} \Delta \vec{W}_{k \varepsilon}\right|_{i, j+1, k} ^{n}+\left.C_{k \varepsilon_{2}} \Delta \vec{W}_{k \varepsilon}\right|_{i, j, k+1} ^{n}\right)
\end{gathered}
$$

\section{5, Diagonally Inverted LU Factorization}

The LU factorization of the $k-\epsilon$ equations produces simple $2 \times 2$ matrix systems which can be inverted algebraically, while the matrix systems associated with the How equations are diagonally inverted using the similarity transformation that produces the following diagonal matrix:

$$
Q^{-1}(A+B+C) Q=\Lambda
$$

which has elements

$$
\begin{gathered}
\lambda_{11}=\lambda_{22}=\lambda_{33}=U+V+W \\
\lambda_{44}=U+V+W-c \sqrt{l_{1}^{2}+l_{2}^{2}+l_{3}^{2}} \\
\lambda_{55}=U+V+W+c \sqrt{l_{1}^{2}+l_{2}^{2}+l_{3}^{2}} \\
\lambda_{i j}=0 \quad \text { when } i \neq j
\end{gathered}
$$

where

$$
\begin{aligned}
q^{2} & =u^{2}+v^{2}+w^{2} \\
l_{1} & =\xi_{x}+\eta_{x}+\zeta_{x} \\
l_{2} & =\xi_{y}+\eta_{y}+\zeta_{y} \\
l_{3} & =\xi_{z}+\eta_{z}+\zeta_{z}
\end{aligned}
$$

and $c$ is the local speed of sound. This similarity transformation, for a local time step defined as:

$$
\Delta t=\frac{C n}{(|A|+|B|+|C|)}
$$

where $C n$ is the Courant number used in the time marching, can be used to transform the lower and upper sweeps into scalar equations with the following vector components $(m=$ $1, \ldots, 5)$

1) Lower Sweep

$$
\begin{gathered}
\left(Q^{-1} \Delta \vec{Y}_{i j k}^{n}\right)_{m}= \\
\frac{\left(\begin{array}{c}
-\Delta t Q^{-1}\left(\left(\bar{\delta}_{\xi}\left(\vec{F}-\vec{F}_{v}\right)+\vec{\delta}_{\eta}\left(\vec{G}-\vec{G}_{v}\right)\right.\right. \\
\left.+\bar{\delta}_{\xi}\left(\vec{H}-\vec{H}_{v}\right)+\vec{T}\right)_{i j k}^{n} \\
\left.-\mu_{i}\left(A_{1} \Delta \vec{Y}_{i-1, j, k}^{n}+B_{1} \Delta \vec{Y}_{i, j-1, k}^{n}+C_{1} \Delta \vec{Y}_{i, j, k-1}^{n}\right)\right)
\end{array}\right)_{m}}{\left(\left(1+\frac{\mu_{1} \beta C, n}{2}\right) I+\frac{\mu \Delta t}{2} \Lambda\right)_{m}}
\end{gathered}
$$

2) Upper Sweep

$$
\left(Q^{-1} \Delta \vec{W}_{i j k}^{n}\right)_{m}=
$$




\section{Steady State Calculations}

The calculation of steady state solutions is made more efficient by using local time-stepping and the multigrid method.

Local time-stepping is used to optimize the time step throughout the flow field. A locally varying time step size, based on a constant Courant number, is used to create a warped time integration that can accelerate the calculation to a steady state without affecting the steady state solution. These time steps are defined identically for both the flow and $k-\varepsilon$ equations, although the value of the Courant numbers used in their evaluation may vary.

The multigrid method is incorporated into the Diagonally Inverted LU scheme to accelerate the removal of low frequency errors from the flow solution and thus increase the efficiency of the time-marching procedure. Following the work of Jameson, ${ }^{16}$ the flow solver is used to smooth out high frequency errors resolvable on any current grid level $(h)$, while the multigrid method is used to eliminate low frequency errors through a sequence of flow calculations on coarser grids $(2 h, 4 h, 8 h, \ldots)$. The multigrid sequencing used is the four-level W- cycle shown below

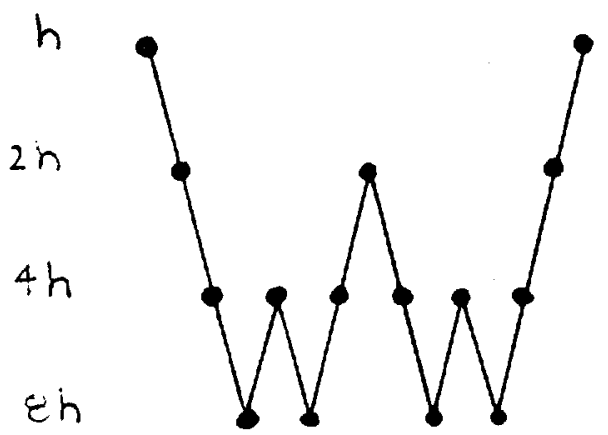

Coarse grid boundary conditions are identical to those used on the fine grid with the exception of the inflow/outflow conditions which are updated only on the fine grids. The Reynolds-Averaged Navier-Stokes equations are solved on the finest grid while only the Euler luxes are evaluated on the coarse grids. Coarse grid residuals are kept smooth by adding only a constant coefficient second difference artifcial dissipation term (the nonlinear blended terms are used only on the fine grid). This treatment attempts to limit the amount of high frequency errors reintroduced into the flow field by the upward interpolation of the coarse grid corrections. The flow solver, in this case the Diagonally Inverted $L U$ scheme, is invoked only once on each grid level and only before transfering the flow field to the next coarser grid. The multigrid cycle defined above requires approximately 1.32 work units of computational effort, where work units are normalized by a single Navier-Stokes calculation on the finest grid. The $k-\varepsilon$ equations are solved only on the finest grid and are not accelerated with the multigrid method. Preliminary attempts at multigridding the $k-\varepsilon$ equations proved to be less efficient than simply solving these equations only on the finest grid.

\section{Results}

Numerical results are presented to illustrate the $\mathrm{Di}$ agonally Inverted LU scheme's ability to calculate threedimensional compressible viscous flows and the convergence acceleration produced by the multigrid method.

Turbomachinery calculations were performed on $\mathrm{H}$ type grids consisting of $96 \times 24 \times 24$ mesh cells in the throughflow, blade-to-blade, and radial directions, respectively. These grids were generated using a modified version of the GRAPE code ${ }^{17}$ originally developed by Sorenson. ${ }^{18}$ All calculations were performed on a CRAY X-MP, where a calculation consisting of 211 work units required approximately 1.5 million words of memory and 40 minutes of CPU time.

The test case used to evaluate the $L U$ scheme is the Annular Cascade designed and extensively tested at NASA Lewis. ${ }^{19,20}$ The computational grid shown in Figure 1 is based on the full annular ring of 36 core turbine stator vanes. The geometry is a $38.10 \mathrm{~mm}$ high untwisted blade of constant profile with an axial chord of $38.23 \mathrm{~mm}$. The stator has a tip diameter of $508 \mathrm{~mm}$ and a 0.85 hub-to-tip radius ratio. Mesh cells found immediately adjacent to solid walls are centered at distances 0.002 of an axial chord away, which correspond to a value of $Y^{+} \approx 60$ for the following flow calculations.

Experimental test conditions of ambient axial inflow and a 0.65 hub-static to inlet-total pressure ratio produce a flow field with mean radius inlet and exit critical velocity ratios of 0.231 and 0.778 respectively. To match the upstream flow conditions (an inflow Mach number of 0.211), the nonrotating ( $R O T=0)$ calculations were run with a 0.665 hub-static to inlet-total pressure ratio $(P R=0.665)$.

Figure 2 shows the convergence history of the Annular Cascade calculation (with multigrid) where a drop of 3.5 orders of magnitude in the flow field error was produced within 200 work units. The residual was reduced from an initial $R E S 1=0.169 \times 10^{1}$ to a final value of $R E S 2=0.572 \times 10^{-3}$ (0.9628 average rate of convergence). A Courant number of $C n=6$ was used in the solution of the fow equations while a $C n=4$ was used in the $k-\varepsilon$ equations (no attempt to optimize these numbers was made).

The resulting flow field is fully subsonic and is compared with experimental data at three spanwise positions. Figures 3,4 , and 5 compare the calculated blade surface static pressure distributions (normalized by inlet total pressure) at $13.3,50$, and 86.7 percent span with the experimental data produced by Goldman and Seasholtz. ${ }^{20}$ The 
[17] Smith, W.A., "Modified Grape Code," Sibley School of Mechanical and Aerospace Engineering FDA Report 87.10, Cornell University, June 1987.

[18] Sorenson, R., “A Computer Program to Generate Two Dimensional Grids About Airfoils and Other Shapes by the use of the Poisson's Equation," NASA TM 81198, 1980.

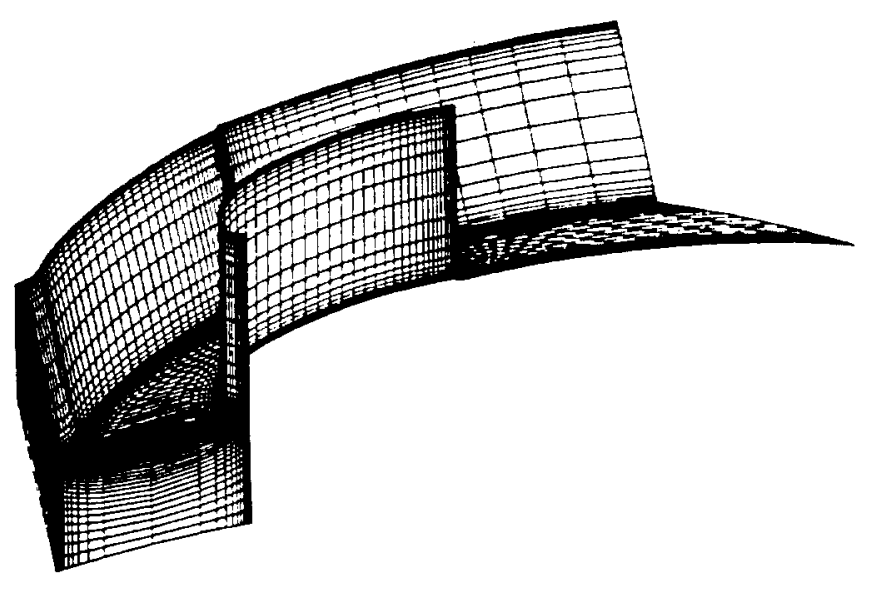

Fig. 1. Computational grid for the Annular Cascade.

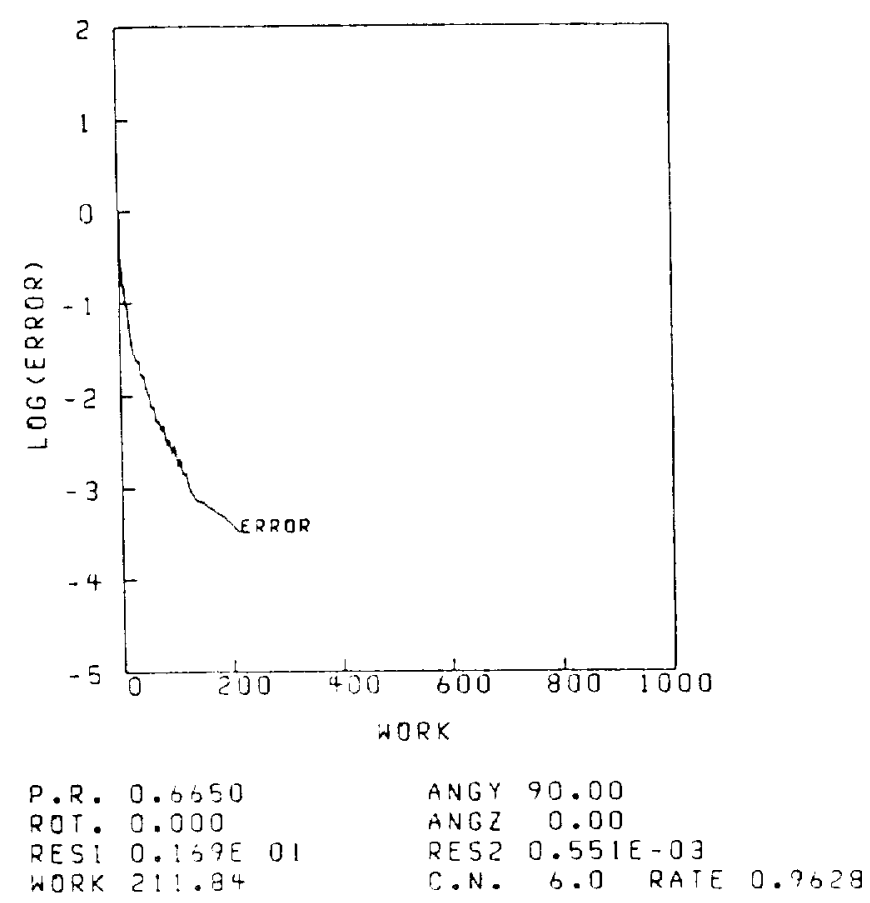

Fig. 2. Convergence history for the multigrid calculation.
[19] Goldman, L.J., and McLallin, K.L., "Cold-Air Annular Cascade Investigation of Aerodynamic Performance of Core-Engine-Cooled Turbine Vanes. 1: Soltd-Vane Performance and Facility Description," NASA TM X$3224,1975$.

(20) Goldman, L.J., and Seasholtz, R.G., "Laser Anemometer Measurements in a Annular Cascade of Core Turbine Vanes and Comparison with Theory," NASA TP. $2018,1982$.

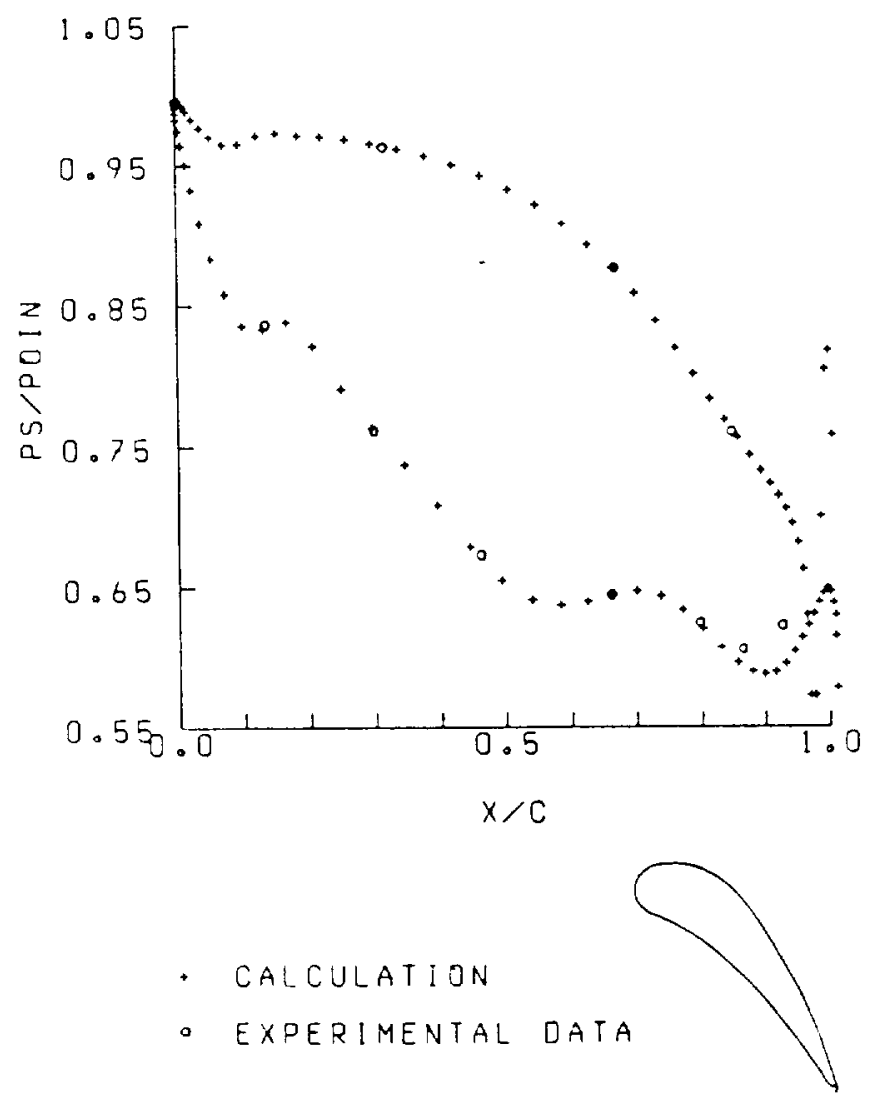

Fig. 3. Static pressure blade distribution at 13.3 percent span. 
